Am. J. Trop. Med. Hyg., 98(3), 2018, pp. 835-837

doi:10.4269/aitmh.17-0199

Copyright $(\odot) 2018$ by The American Society of Tropical Medicine and Hygiene

\title{
Case Report: Family Cluster of Japanese Spotted Fever
}

\author{
Hiroki Matsuura $^{1 *}$ and Kyoko Yokota ${ }^{2}$ \\ ${ }^{1}$ Department of General Internal Medicine, Mitoyo General Hospital, Kanonji, Kagawa, Japan; ${ }^{2}$ Department of Infectious Disease, Kagawa \\ Prefectural Central Hospital, Takamatsu, Kagawa, Japan
}

\begin{abstract}
Spotted fever group rickettsioses are transmitted by several types of arthropods (including ticks, chiggers, fleas, and lice) and are distributed worldwide. Japanese spotted fever (JSF) was discovered as an emerging rickettsiosis in 1984. The annual number of cases has increased 3-fold during the last decade. In Japan, JSF has been mainly reported in an area with warm climate that borders the Pacific Ocean. We describe a family/neighborhood cluster of three cases of JSF in an area of Japan that had previously not been considered endemic.
\end{abstract}

\section{INTRODUCTION}

Spotted fever group rickettsioses are transmitted by several types of arthropods (including ticks, chiggers, fleas, and lice) and are distributed worldwide. ${ }^{1}$ Japanese spotted fever (JSF) was discovered as an emerging rickettsiosis on the island of Shikoku in southwestern Japan in 1984. Since Mahara et al. ${ }^{2}$ first reported a clinical case of JSF in the Tokushima prefecture, a total of 3,000 cases of JSF were reported by the National Institutes of Health in Japan. The annual number of cases has increased 3-fold during the last decade. ${ }^{3}$ In Japan, JSF has been mainly reported in an area with warm climate that borders the Pacific Ocean. Approximately $90 \%$ of reported cases occurred in western Japan. Rickettsiosis is considered as a sporadic disease; however, family clusters and geographical hyperendemicity have been reported. ${ }^{4}$ We experienced three cases of JSF as a family/neighborhood cluster in an area of Japan that had previously not been considered endemic.

\section{CASE REPORTS}

Case 1. A 74-year-old woman was transferred to our hospital with a 4-day history of fever, rash, confusion, and disorientation. She had a temperature of $39.6^{\circ} \mathrm{C}$, blood pressure of $108 / 63 \mathrm{~mm}$ of $\mathrm{Hg}$, respiratory rate of 22 breaths/minutes, and pulse of $121 /$ minutes. Physical examination revealed an eschar on the left lower thigh (Figure 1A). Based on a tentative diagnosis of rickettsiosis, scrub typhus (Tsutsugamushi disease), or JSF, intravenous minocycline was started, Ciprofloxacin hydrochloride was added as a severe case of JSF.

The woman's family history revealed that her husband had been admitted to the same hospital 3 days earlier; he died on the day of her admission. Blood and biopsy samples of the eschar were sent to the local reference laboratory. Polymerase chain reactions (PCRs) of both samples were positive for Rickettsia japonica. (The primary PCR was performed with the R1/R2 [5'-TCAATTCACAACTTGCCATT-3'/5'-TACAAAATTC TAAAAACC-3'] primer combination to detect spotted fever group Rickettsia and a secondary PCR was performed with the Rj5/Rj10 [5'-CGCCATTCTACGTTACTACC-3' /5' -ATTC TAAAAACCATATACTG-3'] primer combination that specifically amplifies $R$. japonica ${ }^{5}$ ). The JSF diagnosis was confirmed through paired serum testing using immunofluorescent (IIF)

*Address correspondence to Hiroki Matsuura, Department of GIM, Mitoyo General Hospital, 708, Himehama, Toyohama-cho, Kanonji-city, Kagawa 769-1695, Japan. E-mail: superonewex0506@yahoo.co.jp assays. Serum antibody titers against $R$. japonica were immunoglobulin $\mathrm{M}(\mathrm{lgM})<40$ and immunoglobulin $\mathrm{G}(\mathrm{IgG})<40$ during the acute period (day 2); titers increased to $\lg M 1: 640$ and IgG 1:320 during the recovery period (day 21). Despite the administration of appropriate antibiotic treatment, we observed a progression of disseminated intravascular coagulation (DIC) in the patient. Therefore, she was transferred to an intensive care unit for 6 days. The patient was treated with minocycline and ciprofloxacin for 10 days. During the clinical course, the patient developed sudden hypotension and tachycardia with a stat hemoglobin of $6.5 \mathrm{mg} / \mathrm{dL}$. Enhanced abdominal computed tomography (CT) revealed a large retroperitoneal hematoma and extravasation from the iliolumbar arteries, which were treated by interventional radiological embolization. The patient was discharged after 74 days and made a full recovery after outpatient rehabilitation.

Case 2 (the patient's husband). An 80-year-old man presented to the hospital with a 5-day history of fever, a papular rash on the extremities and trunk, and an altered mental status. His temperature was $39.6^{\circ} \mathrm{C}$, blood pressure $105 / 47 \mathrm{~mm}$ of $\mathrm{Hg}$, respiratory rate 24 breaths/minutes, and pulse 140/minutes. Chest and abdominal CT showed no abnormalities. Laboratory tests revealed thrombocytopenia, elevated liver enzyme levels, elevated fibrinogen degradation product levels, and activated partial thromboplastin time and prothrombin time. The patient was suspected of having sepsis, resulting in DIC and multiple organ failure. Because the patient presented with mental disturbance, antibiotics and antiviral agents (ceftriaxone, vancomycin, and acyclovir) were administered to cover bacterial meningitis and viral encephalitis; however, these medications were completely ineffective. Three days after the start of treatment, the patient died. After the diagnosis of case 1, he was reexamined; an eschar that had previously not been detected was seen on his left popliteal fossa (Figure 1B). Blood and biopsy samples of the eschar were sent to the local reference laboratory. PCRs of both samples were positive for $R$. japonica. The JSF diagnosis was confirmed with single serum testing using IIF assays antibody titers against $R$. japonica of $\lg M<80$ and $\lg G<80$.

Although she disclosed that they were hobby farmers and lived near grassy areas where ticks might have been present, rickettsiosis had never been reported in these areas. One month later she was discharged, her neighbor was transferred to the hospital and diagnosed with JSF through PCR of a serum sample and paired serum testing using IIF assays antibody titers against $R$. japonica. 


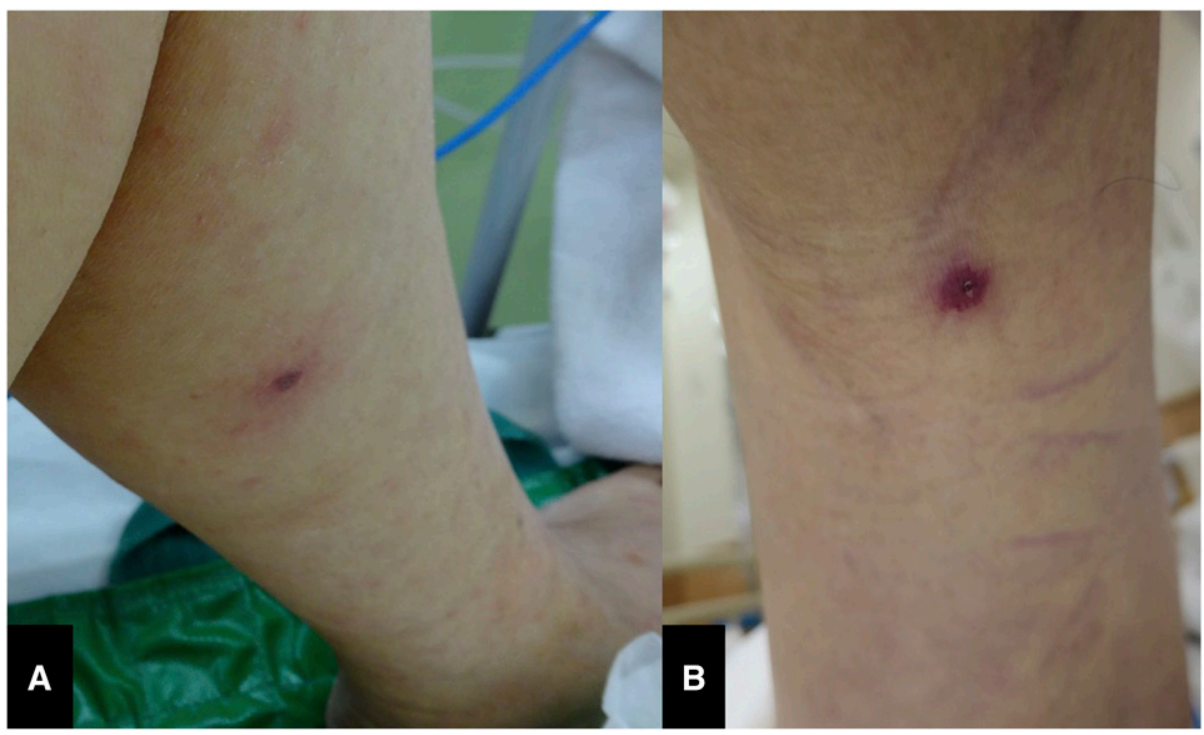

FIGURE 1. (A) Photograph of the eschar on the left lower thigh (case 1). (B) Photograph of the eschar on the left popliteal fossa (case 2). This figure appears in color at www.ajtmh.org.

\section{DISCUSSION}

JSF caused by R. japonica is a relatively newer type of rickettsiosis. Similar to other spotted fever group rickettsioses, many fatal cases have been reported due to a delay in initiating appropriate antibiotic treatment. The overall mortality rate is approximately $5 \%$; those who present late in the illness ( 6 days) have an elevated rate for complications, including myocarditis, ${ }^{6}$ hepatic dysfunction, ${ }^{7}$ acute renal failure, encephalitis, and DIC. ${ }^{8,9}$ In case 1 , the patient's condition deteriorated, although minocycline was started immediately after admission. Her clinical course of 4 days was too short to develop any complication. Complications of JSF tend to occur in the elderly. The patient might not have reported the correct duration of symptom due to her impaired mental status at admission. Recently, immunohistochemical staining for the Rickettsia antigen has been investigated as a tool for an early diagnosis of JSF. However, several studies have indicated that the PCR method using eschar samples is the most sensitive and specific method for an instant and accurate diagnosis of JSF. ${ }^{10}$ Aminoglycosides and $\beta$-lactam antibiotics (penicillin and cephalosporins), which are commonly used in the empirical treatment of febrile diseases, are ineffective in the treatment of JSF; however, minocycline and doxycycline have been found to be effective. Interestingly, a combination therapy of minocycline and new quinolones has been reported to be effective for complicated JSF case. ${ }^{11}$ Prompt and adequate antibiotic treatment of patients suspected of having JSF is often necessary as patients typically do not have defined symptoms, and no rapid diagnostic test is available.

Kagawa prefecture is located on the island of Shikoku and is bordered by Tokushima prefecture. In Kagawa prefecture, nine cases of JSF have been reported since 1984. These cases occurred in the eastern part of Kagawa prefecture that is close to Tokushima prefecture. In this report, all three patients lived in the western part of the Kagawa prefecture, $80 \mathrm{~km}$ away from the eastern part. The first case of JSF in Kagawa prefecture was reported in 2013; before the cases reported herein, only nine cases had been reported in the eastern area of the prefecture. However, in 2008, before the first human case was reported, the Kagawa prefectural environmental research center reported the seroprevalence of $R$. japonica through IIF assays among dogs. This study showed that dogs were highly exposed to $R$. japonica infections, with a seroprevalence ranging from $33.3 \%$ to $56.1 \% .^{12}$ Several studies reported that the seroprevalence of spotted fever group Rickettsiae in autochthonous animals (capybaras, ${ }^{13}$ equines, ${ }^{14}$ deer, ${ }^{15}$ and boars ${ }^{16}$ ) predicted an imminent and high risk for transmitting spotted fever group Rickettsiae from mediators to humans. Therefore, JSF might have been endemic in this area. JSF is often missed by medical providers and healthcare personnel. Most patients do not exhibit the classic features of JSF on their first visit to a medical institution. For example, the rash often appears several days after the onset of fever and may evolve into petechiae. ${ }^{17}$ The absence of a known tick bite is also common and should not deter clinicians from suspecting JSF. A very small number of patients in these reports recalled suffering a tick bite before the onset of illness, although all lived near forested or grassy areas where ticks may have been present. Clinician must, therefore, carefully examine febrile patients with a strong suspicion of rickettsioses in endemic areas. Although few cases of familial clusters of JSF have been reported in Japan, ${ }^{4}$ several outbreaks of Rocky Mountain spotted fever, ${ }^{18,19}$ Mediterranean spotted fever, ${ }^{20}$ and Brazilian spotted fever ${ }^{21}$ have been confirmed. Therefore, as for other spotted fever group rickettsioses, when a case of JSF is suspected, clinicians must pay attention to other habitants who were exposed to the same environment. Multiple cases of infections may occur at the same time, as demonstrated in the current case. Education for medical providers and healthcare personnel is essential. Moreover, it is exceedingly important to promote educational campaigns that emphasize the prevention of tick bites and avoiding exposure to tick habitats. 
Received March 14, 2017. Accepted for publication December 6, 2017.

Published online January 22, 2018.

Authors' addresses: Hiroki Matsuura, Department of General Internal Medicine, Mitoyo General Hospital, Kanonji, Kagawa, Japan, E-mail: superonewex0506@yahoo.co.jp. Kyoko Yokota, Department of Infectious Disease, Kagawa Kenritsu Chuo Byoin, Takamatsu, Kagawa, Japan, E-mail: kyoyoko@med.kagawa-u.ac.jp.

\section{REFERENCES}

1. Parola $P$, Paddock CD, Raoult D, 2005. Tick-borne rickettsioses around the world: emerging diseases challenging old concepts. Clin Microbiol Rev 18: 719-756.

2. Mahara F et al., 1985. The first report of the rickettsial infections of spotted fever group in Japan: three clinical cases. Jpn J Infect Dis 59: 1165-1172.

3. National Institute of Infectious Diseases (JP). NESID Annual Surveillance Data (Notifiable Diseases) 2014-2 [Internet]. Available at: http://www.nih.go.jp/niid/en/survei/2085-idwr/ydata/6058report-ea2014-20.html. Accessed June 16, 2017.

4. Kubozono T, Honda T, 2006. Simultaneous development of Japanese spotted fever in a married couple in Kagoshima Prefecture. Kansenshogaku Zasshi 80: 432-435.

5. Furuya Y, Katayama T, Yoshida T, Kaiho I, 1995. Specific amplification of Rickettsia japonica DNA from clinical specimens by PCR. J Clin Microbiol 33: 487-489.

6. Fukuta Y, Mahara F, Nakatsu T, Yoshida T, Nishimura M, 2007. A case of Japanese spotted fever complicated with acute myocarditis. Jpn J Infect Dis 60: 59-61.

7. Nomura T, Fujimoto T, Ebisutani C, Horiguchi H, Ando S, 2007. The first fatal case of Japanese spotted fever confirmed by serological and microbiological tests in Awaji Island, Japan. Jpn J Infect Dis 60: 241-243.

8. Iwasaki I, Mahara F, Takada N, Fujita H, Ueda T, 2001. Fulminant Japanese spotted fever associated with hypercytokinemia. J Clin Microbiol 39: 2341-2343.

9. Nakata R, Motomura $M$, Tokuda $M$, Nakajima $H$, Masuda $T$, Fukuda T, Tsujino A, Yoshimura T, Kawakami A, 2012. A case of Japanese spotted fever complicated with central nervous system involvement and multiple organ failure. Intern Med 51: 783-786.

10. Kurokawa I, Kondo M, Akachi S, 2013. Early diagnosis of Japan spotted fever by PCR using skin samples. $J$ Infect Chemother 19: 628-632.

11. Mahara F, Miyamoto K, Fujita H, Mutsuda T, 2014. Clinical usefulness of combination therapy with minocycline and ciprofloxacin as a treatment for fulminant cases of Japanese spotted fever. Clin Microbiol 3: 176.

12. Komoda H, Miki K, 2012. About the infiltration situation of Japanese spotted fever in Kagawa Prefecture (Japanese). J Dermatol 11: 2306.

13. Labruna MB, 2009. Ecology of Rickettsia in South America. Ann NY Acad Sci 1: 156-166.

14. Souza CE, Camargo LB, Pinter A, Donalisio MR, 2016. High seroprevalence for Rickettsia rickettsii in equines suggests risk of human infection in silent areas for the Brazilian spotted fever. PLoS One 11: e0153303.

15. Jilintai, Seino N, Matsumoto K, Hayakawa D, Suzuki M, Hata H, Kondo S, Yokoyama N, Inokuma H, 2008. Serological and molecular survey of Rickettsial infection in cattle and sika deer in a pastureland in Hidaka District, Hokkaido, Japan. Jpn J Infect Dis 61: 315-317.

16. Ortuño A, Quesada M, López-Claessens S, Castellà J, Sanfeliu I, Antón E, Segura-Porta F, 2007. The role of wild boar (Sus scrofa) in the eco-epidemiology of $R$. slovaca in northeastern Spain. Vector Borne Zoonotic Dis 7: 59-64.

17. Rapini RP, Bolognia JL, Jorizzo JL, 2007. Dermatology: 2-Volume Set. St. Louis, MO: Mosby, 1130. ISBN 1-4160-2999-0.

18. Jones TF, Craig AS, Paddock CD, McKechnie DB, Childs JE, Zaki SR, Schaffner W, 1999. Family cluster of Rocky Mountain spotted fever. Clin Infect Dis 28: 853-859.

19. Centers for Disease Control and Prevention (CDC), 2004. Fatal cases of Rocky Mountain spotted fever in family clusters-three states, 2003. MMWR Morb Mortal Wkly Rep 21: 407-410.

20. Keysary A, Potasman I, Itzhaki A, Finkelstein R, Yitzhaki S, Strenger C, Rzotkiewicz S, Leitner M, 2007. Clusters of Mediterranean spotted fever in Israel. Vector Borne Zoonotic Dis 7: 143-146.

21. Galvão MA, Dumler JS, Mafra CL, Calic SB, Chamone CB, Cesarino, Filho G, Olano JP, Walker DH, 2003. Fatal spotted fever rickettsiosis, Minas Gerais, Brazil. Emerg Infect Dis 9: 1402-1405. 